\title{
Pelvic floor muscle function in a general population of women with and without pelvic organ prolapse
}

\author{
Marijke Slieker-ten Hove • Annelies Pool-Goudzwaard • \\ Marinus Eijkemans • Regine Steegers-Theunissen • \\ Curt Burger • Mark Vierhout
}

Received: 15 April 2009 /Revised: 8 October 2009 /Accepted: 21 October 2009/Published online: 21 November 2009

(C) The Author(s) 2009. This article is published with open access at Springerlink.com

\begin{abstract}
Introduction and hypothesis This study aims to examine the relationship between pelvic floor muscle function (PFMF) and pelvic organ prolapse (POP) in a general female population.

Methods Cross-sectional study on women aged 4585 years. Validated questionnaires were used to assess pelvic floor muscle function. POP and PFMF were evaluated with vaginal examination. For statistical analysis chi-squared test for trend and analysis of variance were used.

Results Response rate to the questionnaire was $62.7 \%$ $(1,869 / 2,979)$. No significant differences were found in muscle strength and endurance during voluntary muscle contraction between the POP stages. Women with POP stages I and II were significantly less able to achieve
\end{abstract}

M. Slieker-ten Hove · R. Steegers-Theunissen · C. Burger Department of Obstetrics and Gynaecology, Erasmus MC University Medical Centre,

Rotterdam, the Netherlands

A. Pool-Goudzwaard

Department of Neuroscience, Erasmus MC,

University Medical Centre,

Rotterdam, the Netherlands

M. Eijkemans

Department of Public Health, Erasmus MC,

University Medical Centre,

Rotterdam, the Netherlands

R. Steegers-Theunissen

Department of Epidemiology,

Erasmus University Medical Centre,

Rotterdam, the Netherlands effective involuntary muscle contraction during coughing (38.3\% and 37.7\%) than women without POP (75.2\%). Conclusion Involuntary contraction of the PFM during coughing (that resulted in stabilization of the perineum) was significantly weaker in the women with POP stage I and II than in the women without POP.

Keywords Pelvic floor musculature · Pelvic floor muscle function · Pelvic organ prolapse · Involuntary contraction . Intra-abdominal pressure

\section{Introduction}

Pelvic organ prolapse (POP) is a common condition that affects around $75 \%$ of women of all ages and parity, but it
R. Steegers-Theunissen

Department Paediatrics/Paediatric Cardiology, Erasmus MC,

University Medical Centre,

Rotterdam, the Netherlands

R. Steegers-Theunissen

Department Clinical Genetics, Erasmus MC,

University Medical Centre,

Rotterdam, the Netherlands

M. Vierhout

Department of Obstetrics and Gynaecology,

Radboud University Nijmegen Medical Centre,

Nijmegen, the Netherlands

M. Slieker-ten Hove $(\square)$

Softwareweg 5,

3821 BN Amersfoort, the Netherlands

e-mail: m.slieker@somt.nl 
is not always symptomatic [1]. By the age of 79 years, women have an $11 \%$ chance of undergoing at least one surgical treatment for incontinence or POP, with a $29.2 \%$ risk of recurrent surgery [2]. The medical cost of POP is high (more than one billion dollars a year in the USA) [3] and is expected to increase due to global aging. We are therefore faced with the challenge of obtaining knowledge about its pathophysiology in order to achieve adequate prevention or treatment [4].

POP has been attributed to a decrease in pelvic floor muscle support that results in widening of the genital hiatus and stretching of the connective tissue, which enables downward movement of the pelvic organs [5].

Known risk factors for POP are parity, family history, surgery for urinary incontinence (UI) or advanced stage POP, overweight, menopause, race, medication, heavy lifting, increased chronic abdominal pressure caused by heavy physical work or lung disease and smoking, $[1,6]$. Unfortunately, it is not easy to prevent exposure to most of these risk factors.

Although surgery is the preferred treatment, the recurrence versus no recurrence of POP after surgical repair reported in one study was $34.6 \%$ [7]. It was associated with reduced strength of the levator ani $(35.8 \%$ versus $0 \%, p=0.017)$ and a genital hiatus of $5 \mathrm{~cm}$ or larger $(44.2 \%$ versus $27.8 \%)$. Therefore, the question arises as to whether surgery is the optimal and only option to treat POP. As its pathogenesis starts with loss of support of the pelvic floor musculature (PFM), training these muscles might be an effective conservative treatment for POP. Support for muscle training was found in a pilot randomized controlled trial by Hagen et al. [8] in which the women with POP stages I and II showed a decrease in POP symptoms and objective and subjective improvement. The PFM training protocol not only included strength and endurance, but women also were taught to voluntary contract the PFM prior to activities such as coughing in order to train the involuntary muscle response.

It is not clear whether this training protocol is the optimal answer to conservative treatment. However, trials are currently underway. Knowledge is still lacking on PFM functioning (PFMF) in women with POP. Only one study has been performed on the analysis of PFM activity in women with POP prior to corrective surgery [9]. They found a significant relation between weak PFM and severe POP in a multicentre trial. However, they did not use the standardized PFM terminology as put forward by the International Continence Society (ICS) [10]. Especially the assessment of the involuntary contractions of the PFM was lacking.

In order to design an optimal training program for the PFM in women with POP, knowledge is required on the relation between PFMF (muscle strength, endurance assessed by voluntary muscle contraction, and coordination assessed by involuntary muscle contraction) and POP stage in a general female population. Therefore, the aims of our study were to determine:

1. the relation between POP and PFMF in women with and without POP symptoms of vaginal bulging

2. the relation between POP stage and PFMF using the traditional POPQ measurements and a recently developed ICS PFM assessment tool including both voluntary and involuntary PFM contractions[11]

\section{Material and methods}

A cross-sectional study was performed on a general population of mostly white women, aged 45 to 85 years, living in a Dutch town. Figure 1 presents the flowchart of the study.

The total population of women in this age range $(n=$ 2,979 out of 16,000 citizens), registered on the lists of eight out of the nine general practices in the town of Brielle (near Rotterdam, The Netherlands), were approached to participate in the present study. As all the inhabitants are obliged to register with a general practitioner, the study population included $95 \%$ of the women in this age range in the town. Five percent of the female population was missing since one practitioner did not participate. The women were sent information about the study and they could enroll by filling out an informed consent form. They were offered three options: to sign a refusal form, to fill out the questionnaire only, or to fill out the questionnaire and undergo vaginal examination.

After receiving the informed consent the women who agreed to participate were asked to complete a self-report questionnaire. Non-responders received a reminder 8 weeks later that contained the same questionnaire. The data were collected anonymously. To encourage a high response to the questionnaire, we used envelopes with the name and logo of the Erasmus University, colored paper, and stamped-addressed return envelopes [12]. Each reminder included the same questionnaire. To investigate selection bias, non-responders to the reminders were invited to complete a short questionnaire that comprised five questions about: age, parity, presence of stress urinary incontinence (yes/no), fecal incontinence (yes/no), and feeling of vaginal bulging (yes/no).

The questionnaires used in this study combined several validated Dutch versions of pelvic floor questionnaires, such as the Urogenital Distress Inventory [13] and the Defecation Distress Inventory [14]. Data extracted from the questionnaires, presented in this article, included body mass index (BMI), ethnicity, educational level, parity, menopaus- 
Fig. 1 Flowchart of the study

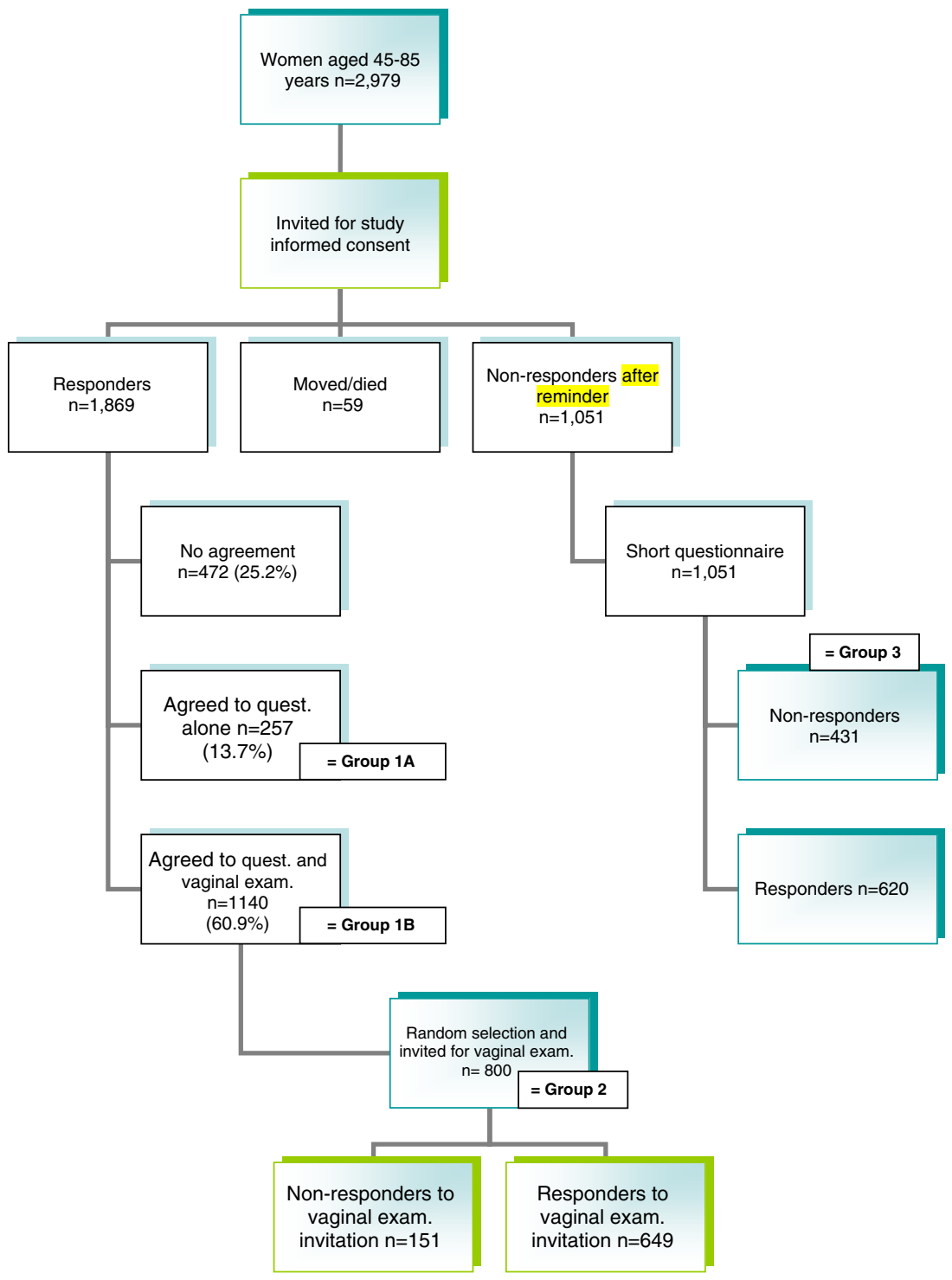

al status, hormone replacement therapy, smoking, previous pelvic floor surgery, family history, and heavy physical work undertaken currently or in the past. Women were defined as postmenopausal when their last menstruation had occurred at least 12 months previously. Women were defined symptomatic for prolapse when they answered positive on the question 'do you see and/or feel a vaginal bulge?'

\section{Vaginal examination}

From the group of participants who gave informed consent to undergo vaginal examination ( $n=1,140$, group 1B), 800 women were randomly selected for PFMF assessment and POPQ measurement (group 2). (All the response forms were coded with a number that identified the women's age and then a research assistant selected the study sample at random by age). All the vaginal examinations were performed at the women's own general practice in the spring period of 2004. The women were asked to empty their bladder before the examination and were then placed on the examination table in the lithotomy position, with a slightly elevated body and the legs bent to about $30^{\circ}$ of flexion.

Table 1 presents the PFMF assessment method, the criteria, and verbal instructions. Voluntary muscle contraction (VMC) and effective involuntary muscle contraction (IMC) during coughing, relaxation, and straining were scored by visual inspection and digital palpation. Data were also obtained using the reliable and valid POPQ measurement method, developed by the ICS [15].

One gynecologist and one physiotherapist performed all the vaginal examinations. Each participant was assessed once. A great deal of attention was paid to training the two examiners before starting the study. They practiced the vaginal examination protocol until $100 \%$ agreement was reached between their scores. This process was performed 
Table 1 PFM assessment with outcome and outcome measures between parenthesis: verbal instruction

\begin{tabular}{|c|c|c|}
\hline & Outcome & Outcome measures \\
\hline \multicolumn{3}{|l|}{ Visual inspection } \\
\hline \multirow[t]{2}{*}{ Co-contraction visible } & Yes & Any co-activity of muscles other than $\operatorname{Tr} A b$ \\
\hline & No & No co-activity of other muscles visible \\
\hline \multirow[t]{2}{*}{ Incontinence during coughing } & Yes & \\
\hline & No & \\
\hline \multicolumn{2}{|c|}{ Palpation during voluntary muscle contraction } & Abbreviation: VMC \\
\hline \multicolumn{3}{|c|}{ ('Lift and squeeze your PFM' and if this was not helpful enough, 'try to avoid loss of urine or flatus') } \\
\hline \multirow[t]{2}{*}{ Urethral lift } & Yes & Any urethral lift palpable \\
\hline & No & No urethral lift is palpable \\
\hline \multirow[t]{3}{*}{ Inward movement perineum } & Yes & Any inward movement of the perineum \\
\hline & No & No inward movement of the perineum \\
\hline & Downward & Any downward movement of the perineum \\
\hline \multirow[t]{2}{*}{ Levator closure } & Yes & Any levator closure movement palpable \\
\hline & No & No levator closure movement palpable \\
\hline \multirow[t]{3}{*}{ Voluntary relaxation } & Complete & Direct beyond rest level \\
\hline & Partly & Direct relaxation to rest level \\
\hline & Absent & No relaxation palpable \\
\hline \multirow{4}{*}{$\begin{array}{l}\text { Maximal voluntary contraction ('lift and squeeze your PFM as } \\
\text { hard as possible') }\end{array}$} & Strong & Strong closure and lifting cranio-anterior movement palpable \\
\hline & Normal & Closure and lifting cranio-anterior movement palpable \\
\hline & Weak & Short contraction, no closure palpable \\
\hline & Absent & No contraction \\
\hline Endurance & 1 to 10 & \\
\hline \multicolumn{3}{|c|}{ ('Make a steady but firm contraction and hold it as long as you can, while repeating hold and hold and hold') } \\
\hline $\begin{array}{l}\text { Palpation during involuntary muscle contraction during } \\
\text { coughing ('cough forcefully') }\end{array}$ & & Abbreviation: IMC \\
\hline \multirow[t]{2}{*}{ Movement perineum } & Yes & $\begin{array}{l}\text { No or ineffective contraction that allows any downward } \\
\text { movement of the perineum }\end{array}$ \\
\hline & No & $\begin{array}{l}\text { Effective IMC that results in no downward movement of the } \\
\text { perineum }\end{array}$ \\
\hline \multirow[t]{2}{*}{ During straining ('give a strong push') involuntary relaxation } & Yes & Any downward movement of the perineum \\
\hline & No & No downward movement \\
\hline
\end{tabular}

$V M C$ voluntary muscle contraction, $I M C$ involuntary muscle contraction

at the Pelvic Floor Centre of the Erasmus Medical Centre, Rotterdam, The Netherlands. The two examiners were blinded to the results of the questionnaire. The Medical Ethics Research Committee (METC) of the Erasmus Medical Centre approved this study.

\section{Statistical analysis}

Individual PFM items that consisted of yes/no scores or three category scores were compared in relation with the five POPQ stages and the symptom of vaginal bulging in two categories, by means of chi-square tests and tests for trends. Analysis of variance (ANOVA), testing for a difference across response categories, was used to compare the outcome on the PFM item endurance (scored as continuous variable). The level of significance was set at $p<0.05$. All the analyses were performed using the Statistical Package for Social Science (SPSS Inc) 15.0.

\section{Results}

Response rate

The response rate to the questionnaire was $62.7 \%(1869 /$ 2979). In the group of 1,869 responders, $472(25.2 \%)$ women refused to participate, 257 (13.7\%) women (group $1 \mathrm{~A}$ in Table 2) agreed to fill out the large questionnaire only, and $1,140(60.9 \%)$ agreed to fill out the questionnaire and undergo vaginal examination (group 1B). Eight hundred out of the 1,140 women were selected at random and sent an invitation to undergo vaginal examination 
Table 2 Demographic characteristics of the total study population and those who underwent vaginal examination expressed as numbers (\%) and means

\begin{tabular}{|c|c|c|}
\hline $\begin{array}{l}\text { Characteristics of the } \\
\text { total study population }\end{array}$ & $\begin{array}{l}\text { Questionnaire } \\
\text { (total groups } \\
1 \mathrm{~A} \text { and } 1 \mathrm{~B} \text { ) } \\
n=1,397^{\mathrm{c}}\end{array}$ & $\begin{array}{l}\text { Vaginal exam. } \\
\text { (total group 2) } \\
n=649\end{array}$ \\
\hline $\begin{array}{l}\text { Mean age } \\
\text { (range } 45-84 \text { years) }\end{array}$ & $58.0(\mathrm{SD} \pm 9.2)$ & $58.3(\mathrm{SD} \pm 9.0)$ \\
\hline \multirow[t]{2}{*}{ Mean BMI } & $n=1,364$ & $n=634$ \\
\hline & $25.6(\mathrm{SD} \pm 3.9)$ & $25.6(\mathrm{SD} \pm 3.7)$ \\
\hline Race & $n=1,372$ & $n=640$ \\
\hline White & $1,351(96.7)$ & $632(97.4)$ \\
\hline \multirow[t]{2}{*}{ Non-white } & $20(1.4)$ & $8(1.2)$ \\
\hline & $n=1340$ & $n=639$ \\
\hline Median parity & 2 & 2 \\
\hline 0 & $120(8.6)$ & $49(7.6)$ \\
\hline 1 & $215(15.4)$ & $86(13.3)$ \\
\hline 2 & $675(48.3)$ & $321(49.5)$ \\
\hline$\geq 3$ & $387(27.7)$ & $183(28.2)$ \\
\hline Educational level & $n=1,374$ & $n=634$ \\
\hline Primary only & $139(9.9)$ & $70(11.1)$ \\
\hline Intermediate & 1,039 (75.6) & $480(75.7)$ \\
\hline Higher & $196(14.3)$ & $84(13.2)$ \\
\hline Menopausal status & $n=1,383$ & $n=645$ \\
\hline Premenopausal & $374(26.8)$ & $167(25.7)$ \\
\hline Postmenopausal $^{\mathrm{a}}$ & $1,009(72.2)$ & $478(73.7)$ \\
\hline Smoking & $n=1,382$ & $n=642$ \\
\hline Current smoker & $280(20)$ & $134(20.6)$ \\
\hline Surgical history & $n=1,384$ & $n=645$ \\
\hline \multirow[t]{2}{*}{ Prolapse } & $103(7.4)$ & $54(8.3)$ \\
\hline & $n=1,382$ & $n=643$ \\
\hline \multirow[t]{2}{*}{ Incontinence } & $47(3.4)$ & $24(3.7)$ \\
\hline & $n=1,383$ & $n=643$ \\
\hline Hysterectomy & $234(16.8)$ & $109(16.8)$ \\
\hline Family history & $n=985$ & $n=441$ \\
\hline \multirow[t]{2}{*}{ Mother POP } & $359(25.7)$ & $161(36.5)$ \\
\hline & $n=870$ & $n=398$ \\
\hline Mother UI & $258(29.7)$ & $122(30.6)$ \\
\hline Heavy physical work ${ }^{\mathrm{b}}$ & $n=1,381$ & $n=642$ \\
\hline \multirow[t]{2}{*}{ Currently } & $269(19.3)$ & $130(20)$ \\
\hline & $n=1,384$ & $n=645$ \\
\hline Ever & $619(44.3)$ & $293(45.1)$ \\
\hline
\end{tabular}

${ }^{a}$ Women whose last menstruation occurred at least 1 year previously

${ }^{\mathrm{b}}$ Heavy physical work: long-term, much lifting and bending

${ }^{\mathrm{c}}$ Due to missing data percentages do not always correspond with the total group

(group 2): 653 women complied (81.6\%). Thus, a total of $46.7 \%$ women who participated in the study underwent the vaginal examination $(649 / 1,397)$. In the non-responder group who did not respond to the first and second requests to participate in the study (group 3), 59\% completed and returned the short questionnaire $(620 / 1,051)$.

Demographic characteristics

Demographic characteristics of the total study population and the different groups (group $1(\mathrm{~A}+\mathrm{B})$ and the vaginal examination group 2) are presented in Table 2. No significant differences in demographic characteristics were found between group 1 (total questionnaire group) and group 3 (the non-responder group who replied to the short questionnaire). In the vaginal examination group, 79 out of the 649 women reported seeing and/or feeling vaginal bulging (scored by the questionnaire). No significant differences were found in the demographic characteristics between these three groups.

PFMF and the POP symptom of vaginal bulging

Table 3 presents the different components of PFMF potentially associated with symptomatic and asymptomatic POP in women who had a vaginal examination. There were no significant differences in any of the PFMF items between women with and women without POP symptoms, except for voluntary relaxation of the PFM that had a significant score $(p=0.011)$. More women who were symptomatic for vaginal bulging had better voluntary relaxation compared to the asymptomatic women $(64.4 \%$ versus $49.7 \%$ )

\section{PFMF versus POPQ}

Table 4 presents the POPQ stage versus the different PFMF items. All the components of PFMF in the women with stage I-IV POP were compared to those in the women without POP and tested for significance. Prevalence of POPQ stage were $25 \%$ (161) for stage $0,36.5 \%$ (235) for stage I, 33\% (212) for stage II, 5\% (32) for stage III, and $0.5 \%$ (3) for stage IV (from six women POPQ data were missing). Visible co-contractions (less often visible in stage 0 than in higher stages), incontinence during coughing (increasing prevalence with increasing stage), and levator closure (decreasing prevalence with increasing stage) differed significantly between the groups (resp. $p=0.006$, $p=0.001$, and $p=0.001)$. POP stage had a significant influence on effective involuntary PFM contraction (IMC) to counteract a sudden increase in intra-abdominal pressure (IAP) during coughing $(75.2 \%$ in stage 0 versus $38.3 \%$ in stage I and $37.7 \%$ in stage II, $p=0.001)$. Significant differences were also found in the caudal movement of the perineum during straining. Women with the lower stages of POP were less able to perform the proper straining technique than the women with $\geq$ stage II. 
Table 3 Pelvic floor muscle function in numbers (percentages) in women who were symptomatic or asymptomatic for POP symptoms

\begin{tabular}{|c|c|c|c|c|}
\hline & Response & Symptomatic for POP $(n=79)$ & Asymptomatic for POP $(n=570)$ & $p$ value $^{\mathrm{a}}$ \\
\hline \multicolumn{5}{|l|}{ Visual inspection } \\
\hline \multirow[t]{2}{*}{ Co-contraction visible } & Yes & $26(33.8)$ & $194(34.8)$ & 0.486 \\
\hline & No & $51(66.2)$ & $364(65.2)$ & \\
\hline \multirow[t]{2}{*}{ Incontinence during coughing } & Yes & $9(11.4)$ & $47(8.4)$ & 0.244 \\
\hline & No & $70(88.6)$ & $513(91.6)$ & \\
\hline \multicolumn{5}{|c|}{ Palpation during voluntary muscle contraction } \\
\hline \multirow[t]{2}{*}{ Urethral lift } & Yes & $45(60)$ & $342(63)$ & 0.352 \\
\hline & No & $201(37)$ & $201(37)$ & \\
\hline \multirow[t]{3}{*}{ Inward movement perineum } & Yes & $63(81.8)$ & $420(76.2)$ & 0.230 \\
\hline & No & $14(18.2)$ & $126(22.9)$ & \\
\hline & Downward & 0 & $5(0.9)$ & \\
\hline \multirow[t]{2}{*}{ Levator closure } & Yes & $45(59.2)$ & $337(62.3)$ & 0.345 \\
\hline & No & $31(40.8)$ & $204(37.7)$ & \\
\hline \multirow[t]{3}{*}{ Voluntary relaxation } & Good & $47(64.4)$ & $259(49.7)$ & \\
\hline & Delayed & $18(24.7)$ & $152(29.2)$ & 0.011 \\
\hline & Incomplete & $8(11)$ & $110(21.1)$ & \\
\hline \multirow[t]{5}{*}{ Voluntary contraction } & Absent & $4(5.1)$ & $29(5.2)$ & 0.592 \\
\hline & Weak & $26(32.9)$ & $175(31.3)$ & \\
\hline & Normal & $47(59.5)$ & $326(58.2)$ & \\
\hline & Strong & $2(2.5)$ & $30(5.4)$ & \\
\hline & & Mean (SD) & Mean (SD) & \\
\hline Endurance & $0-10 \mathrm{~s}$ & $6.25(3.11)$ & $6.25(3.31)$ & 0.987 \\
\hline \multicolumn{5}{|c|}{ Palpation during involuntary muscle contraction while coughing } \\
\hline Downward movement perineum & Yes (ineff. IMC) & $41(51.9)$ & $291(52)$ & 0.543 \\
\hline No downward movement perineum & No (eff. IMC) & $38(48.1)$ & $269(48)$ & \\
\hline \multicolumn{5}{|c|}{ Palpation during involuntary muscle relaxation while straining } \\
\hline Caudal perineal movement during straining & Yes & $73(93.6)$ & $493(89.5)$ & 0.177 \\
\hline No caudal movement & No & $5(6.4)$ & $58(10.5)$ & \\
\hline
\end{tabular}

${ }^{\text {a }}$ Statistical comparison by chi-squared test for trend, except for mean endurance in seconds: $F$ test from ANOVA

\section{Discussion}

This was the first study that tested PFMF in relation to POP in a general female population. We found a significant difference in effective IMC to counteract sudden increases in IAP during coughing between the women without POP and those with different stages of POP. It is difficult to explain why there was such an enormous decrease in the ability to resist IAP in the women with stage I POP. Future research should address whether this decrease is a contributing factor to the development of POP.

The item PFMF levator closure during VMC demonstrated significant differences between the POP stages. We hypothesize that part of the process of POP development next to the demonstrated widening of the genital hiatus [16] can be a weak ability to perform levator closure during an increase in IAP. It is a well-recognized physiological process that during physical activities, people hold their breath and stabilize their diaphragm to create greater strength and consequently, a strong increase in IAP. PFM are reflex activated during any stress. This activation is preset in particular motor patterns such as coughing and is not just a reflex response to increased IAP [17]. Such increases in IAP are present not only during heavy lifting or sports, but also in daily activities and they require optimal IMC. Our study revealed that muscle strength and endurance were not significantly different between the POP stages. Although strength and endurance are necessary to have optimal levator closure, our findings support the assumption that muscle strength and endurance are not the only key issues in the design of new treatment strategies or preventive measures for POP. No significant differences in PFMF were found between the women who reported that they were symptomatic for vaginal bulging and those without these symptoms, except for relaxation after VMC. In one of our earlier publications, vaginal examination 
Table 4 Association between pelvic floor muscle function and pelvic organ prolapse, measured with the POPQ

Outcome

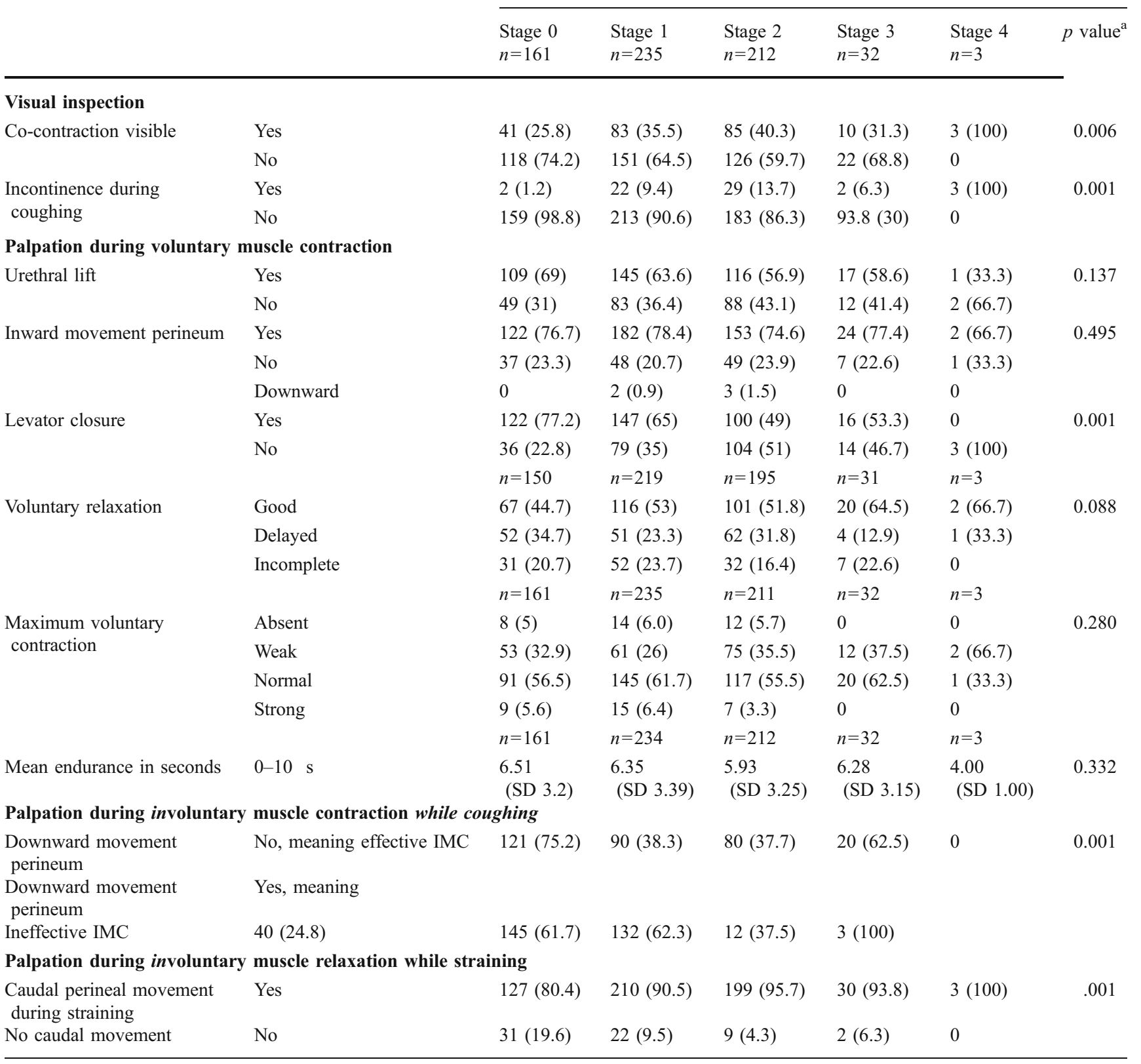

Numbers (percentages)

${ }^{\text {a }}$ Statistical comparison by chi-squared test for trend, except for mean endurance in seconds: $F$ test from ANOVA

revealed that only $30.7 \%$ of the women with POP 'at or beyond the hymen' were symptomatic [18]. This might explain the lack of significant differences in PFMF between the symptomatic group and the asymptomatic group.

Several recommendations can be made for POP treatment and preventive measures based on our study results. Apparently, strong PFM alone does not seem to prevent the development of POP; however, we must be careful making these conclusions due to the character of this study. To study the effect of PFM training on the prevention and treatment of POP, Bo [19] reviewed basic research and case-control studies and put forward two hypotheses: (1) women can learn to contract their PFM consciously before and during an increase in IAP and will continue to make such contractions as a behavioral modification in order to prevent descent of the pelvic contents and (2) women can learn to build up 'muscle tone' and structural support of the pelvic floor through regular strength training over time. Owing to the lack of high-quality randomized controlled trials on the effect of PFM training in women with POP, 
neither of the hypotheses could be confirmed. Longitudinal studies are necessary to provide reliable evidence. Some women might achieve greater PFM strength and endurance as a direct reaction to the development of POP, whereas others might experience deterioration in PFMF due to the progression of POP. It seems that good coordination of the PFM, as demonstrated by effective IMC, is more important. Normal or strong VMC and good PFM endurance do not guarantee effective IMC of the PFM [20]. Therefore, clinicians should test VMC and IMC to gain insight into a woman's ability to counteract any sudden increase in IAP.

Based on the results of the present study, the inclusion of effective IMC of the PFM in new treatment plans gives an indication of a likely effectiveness, as was shown in the recent pilot RCT study by Hagen [8], in which women with stages I and II POP were treated effectively with a protocol that included greater awareness of the location of the PFM and the importance of pre-contraction before an IAP increase, for example when coughing or lifting. In daily practice, it will be challenging to recruit the women without POP and women with stage I so that PFM awareness and training can be started at an early stage in the form of preventive measures. In a recent study, a new combination of risk factors was identified, namely vaginal bulging during pregnancy, a family history of POP, and heavy physical work [6]. The first steps are the organization of antepartum or postpartum training groups and 'POPprevention-groups' for women at risk.

Some reservation must be taken into account when making assumptions about the effect of PFM training to prevent the development or progression of POP. Postpartum MRI and ultrasound studies have demonstrated asymmetrical damage, avulsions, and abnormalities of the pelvic floor [21, 22]. As the pelvic floor is primarily seen as a functional unit (that combines the closure system of the excretory tracts, support system for the pelvic viscera, and a participating unit in the sexual response) it does not seem to be possible to activate the right half or left half of the PFM separately, or to strictly separate activation of individual pairs of muscles [17, 23]. Therefore, it would be worthwhile to study the effect of PFM training in women with asymmetrical damage, in relation to the prevention of POP or other symptoms of pelvic floor dysfunction.

\section{Strengths and limitations}

The strength of our study was the ethnic homogeneity, because almost all of the women were white, which eliminated racial bias from the results. Furthermore, broad data were obtained from a large general study group using a combination of questionnaires and vaginal examination. However, despite the high compliance, selection bias can never be excluded. Another limitation was the lack of blinding of the assessors for the POPQ results. This might cause some bias. However, we regard this possible bias not as a major problem since (a) no interpretation of the results was made during the assessment and (b) the findings of this study were not hypothesized prior to the study.

Another remark can be made regarding the assessment scheme used. Although face validity and reliability of the assessment scheme was tested prior to this study only one study supports the use of this assessment of pelvic floor muscle function. We would recommend further research to demonstrate the value of this assessment scheme in larger populations.

This study was performed on a general population of mainly white women $(98 \%)$ with a mean BMI of 25 . Therefore, extrapolation to other populations has to be performed with caution.

\section{Conclusions}

Women with POP lacked effective IMC of the PFM. More knowledge is needed on the role of PFM training in prevention of POP and the pathogenesis of POP.

Acknowledgement We are very grateful to the women who participated in this study and to Mrs. G.M. Schoenmaker (MD) and her group of general practitioners for their hospitality and cooperation in this study.

Funding Unrestricted grant from NV Organon, Oss, The Netherlands

\section{Conflicts of interest None.}

Open Access This article is distributed under the terms of the Creative Commons Attribution Noncommercial License which permits any noncommercial use, distribution, and reproduction in any medium, provided the original author(s) and source are credited.

\section{References}

1. Swift SE, Tate SB, Nicholas J (2003) Correlation of symptoms with degree of pelvic organ support in a general population of women: what is pelvic organ prolapse? Am J Obstet Gynecol 189 (2):372-377 discussion 377-379

2. Olsen AL, Smith VJ, Bergstrom JO, Colling JC, Clark AL (1997) Epidemiology of surgically managed pelvic organ prolapse and urinary incontinence. Obstet Gynecol 89(4):501-506

3. Subak LL, Waetjen LE, van den Eeden S, Thom DH, Vittinghoff E, Brown JS (2001) Cost of pelvic organ prolapse surgery in the United States. Obstet Gynecol 98(4):646-651

4. DeLancey JO (2005) The hidden epidemic of pelvic floor dysfunction: achievable goals for improved prevention and treatment. Am J Obstet Gynecol 192(5):1488-1495

5. Chen L, Ashton-Miller JA, Hsu Y, DeLancey JO (2006) Interaction among apical support, levator ani impairment, and anterior vaginal wall prolapse. Obstet Gynecol 108(2):324-332 
6. Slieker-Ten Hove MC, Pool-Goudzwaard AL, Eijkemans MJ, Steegers-Theunissen RP, Burger CW, Vierhout ME (2008) Symptomatic pelvic organ prolapse and possible risk factors in a general population. Am J Obstet Gynecol 200(2):184.e1-7 Epub 2008 Dec 25

7. Vakili B, Zheng YT, Loesch H, Echols KT, Franco N, Chesson RR (2005) Levator contraction strength and genital hiatus as risk factors for recurrent pelvic organ prolapse. Am J Obstet Gynecol 192(5):1592-1598

8. Hagen S, Stark D, Glazener C, Sinclair L, Ramsay I (2009) A randomized controlled trial of pelvic floor muscle training for stages I and II pelvic organ prolapse. Int Urogynecol J Pelvic Floor Dysfunct 20(1):45-51

9. Borello-France DF, Handa VL, Brown MB, Goode P, Kreder K, Scheufele LL et al (2007) Pelvic-floor muscle function in women with pelvic organ prolapse. Phys Ther 87(4):399-407

10. Messelink B, Benson T, Berghmans B et al (2005) Standardization of terminology of pelvic floor muscle function and dysfunction: report from the pelvic floor clinical assessment group of the International Continence Society. Neurourol Urodyn 24:374-380

11. Slieker-Ten Hove MC, Pool-Goudzwaard AL, Eijkemans MJ, Steegers-Theunissen RP, Burger CW, Vierhout ME (2009) Face validity and reliability of the first digital assessment scheme of pelvic floor muscle function conform the new standardized terminology of the International Continence Society. Neurourol Urodyn 28(4):295-300

12. Edwards P, Roberts I, Clarke M, Diguiseppi C, Pratap S, Wentz R et al (2002) Increasing response rates to postal questionnaires: systematic review. Bmj 324:1183

13. Van Der Vaart CH, De Leeuw JR, Roovers JP, Heintz AP (2000) [The influence of urinary incontinence on quality of life of community-dwelling, 45-70 year old Dutch women] De invloed van urine-incontinentie op de kwaliteit van leven bij thuiswonende Nederlandse vrouwen van 45-70 jaar. Ned Tijdschr Geneeskd 144:894-897
14. Van Brummen HJ, Bruinse HW, Van De Pol G, Heintz AP, Van Der Vaart CH (2006) Defecatory symptoms during and after the first pregnancy: prevalences and associated factors. Int Urogynecol J Pelvic Floor Dysfunct 17:224-230

15. Bump RC, Mattiasson A, Bo K, Brubaker LP, Delancey JO, Klarskov $P$ et al (1996) The standardization of terminology of female pelvic organ prolapse and pelvic floor dysfunction. Am J Obstet Gynecol 175:10-17

16. Schaffer JI, Wai CY, Boreham MK (2005) Etiology of pelvic organ prolapse. Clin Obstet Gynecol Sep 48(3):639-647

17. Deindl FM, Vodusek DB, Hesse U, Schussler B (1993) Activity patterns of pubococcygeal muscles in nulliparous continent women. Br J Urol 72:46-51

18. Slieker-Ten Hove MC, Pool-Goudzwaard AL, Eijkemans MJ, Steegers-Theunissen RP, Burger CW, Vierhout ME (2009) Prediction model and prognostic index to estimate clinically relevant pelvic organ prolapse in a general female population. Int Urogynecol J pelvic Floor Dysfunct 20:1013-1021

19. Bo K (2006) Can pelvic floor muscle training prevent and treat pelvic organ prolapse? Acta Obstet Gynecol Scand 85(3):263-268

20. Slieker-Ten Hove MC, Pool-Goudzwaard AL, Eijkemans MJ, Steegers-Theunissen RP, Burger CW, Vierhout ME (2009) Pelvic floor muscle function in a general female population in relation with age and parity and the relation between voluntary and involuntary contractions of the pelvic floor musculature. Int Urogynecol J pelvic Floor Dysfunct, September 2009

21. DeLancey JO, Kearney R, Chou Q, Speights S, Binno S (2003) The appearance of levator ani muscle abnormalities in magnetic resonance images after vaginal delivery. Obstet Gynecol 101 (1):46-53

22. Dietz HP, Gillespie AV, Phadke P (2007) Avulsion of the pubovisceral muscle associated with large vaginal tear after normal vaginal delivery at term. Aust N Z J Obstet Gynaecol 47(4):341-344

23. Vereecken RL, Derluyn J, Verduyn H (1975) electromyography of the perineal striated muscles during cystometry. Urol Int 30:92-98 